\title{
La Lente Evolution Du Système Fiscal Marocain : Les Faits Et Les Enseignements
}

\author{
M. Hafid Barka \\ Enseignant-chercheur en Economie-Gestion à l'Institut National de Postes et \\ Télécommunications (INPT-Rabat), Morocco \\ M. Mohammed Saber Hassainate \\ Enseignant-chercheur; Département Gestion, Faculté de Droit Agdal, \\ Université Mohammed V-Rabat, Morocco
}

Doi: 10.19044/esj.2018.v14n4p116 URL:http://dx.doi.org/10.19044/esj.2018.v14n4p116

\begin{abstract}
The present work aims to analyze the evolution of the Moroccan tax system from the independence to the implementation of the tax reform of the 1980s in order to draw the lessons and to be able to decide on its future evolutions. Indeed, fiscal developments and "reforms" succeeded one another since the independence in 1956, with no significant evolution in the Moroccan tax system inherited from the French protectorate. Yet this system was characterized by imbalances; inconsistencies and inequities. A profound tax reform has become inevitable. After an undue delay; The Government has proceeded from 1984 to the reform of the effective tax system with a view to its modernization and simplification. This reform has resulted, in fact, in the passage of a heavy and complex schedular or analytical system, characterized by blatant inequalities, imbalances and inconsistencies to a modern and synthetic taxation system. At the end of this reform; three major synthetic taxes were set up: T.V.A, I.S and I.R. The reform also concerned the taxation of local authorities. With the implementation of the tax reform since 1984, the Moroccan tax system has certainly made progress but limitations related in particular to the architecture of the system and its structure and new synthetic taxes persist. Future research will focus on assessing the tax reforms and adjustments of the years 2000 and the prospects for reforming the Moroccan tax system.
\end{abstract}

Keywords: Moroccan tax system; schedular system; reform; synthetic system; balance sheet 


\section{Résumé}

Le présent travail a pour objectif d'analyser l'évolution du système fiscal marocain de l'indépendance à la mise en œuvre de la réforme fiscale des années 1980 afin d'en tirer les enseignements et pouvoir se prononcer sur ses évolutions futures. En effet, depuis l'indépendance en 1956, les aménagements et "réformes" fiscaux se sont succédés sans que le système fiscal marocain hérité du protectorat français évolue nettement. Pourtant ce système se caractérisait par des déséquilibres, incohérences et injustices. Une réforme fiscale profonde est devenue inévitable. Après un report indu, les pouvoirs publics ont procédé à partir de 1984 à la réforme du système fiscal en vigueur en vue de sa modernisation et sa simplification. Cette réforme s'est traduite, en effet, par le passage d'un système cédulaire ou analytique, lourd, complexe et caractérisé par des inégalités flagrantes, des déséquilibres et des incohérences à un système d'imposition moderne et synthétique. A l'issue de cette réforme, trois grands impôts synthétiques ont été mis en place : T.V.A, I.S et I.R. La réforme a concerné aussi la fiscalité des collectivités locales. Avec la mise en œuvre de la réforme fiscale depuis 1984, le système fiscal marocain a réalisé certes des avancées mais des limites liées notamment à l'architecture du système et sa structure et aux nouveaux impôts synthétiques persistent. De futurs travaux de recherches s'attèleront à évaluer les réformes et aménagements fiscaux des années 2000 et les années suivantes et aux perspectives de réformes du système fiscal marocain.

Mots-clés: Système fiscal marocain, système cédulaire, réforme, système synthétique ; bilan

\section{Introduction}

De l'indépendance en 1956 jusqu'en 1984, le système fiscal marocain en vigueur était hérité du protectorat français. Les responsables marocains ont attendu le milieu des années 1980 pour réformer un système fiscal complexe et aux multiples défaillances. La réforme fiscale des années 1980 constitue un grand tournant dans l'évolution du système fiscal marocain. Elle en a profondément modifié l'architecture générale. Les impôts synthétiques comme la taxe sur la valeur ajoutée (TVA), l'impôt sur les sociétés (IS) et l'impôt général sur le revenu (IGR) se sont substitués aux impôts cédulaires en vigueur. La mise en œuvre de la réforme fiscale des années 1980 s'est accompagnée d'une série d'aménagements qui ont débouché notamment sur la réduction des taux d'imposition, l'élargissement de l'assiette fiscale, la réduction de la charge fiscale et la simplification de la gestion fiscale. Deux autres dates charnières dans l'évolution du système fiscale marocain sont à souligner : les assises fiscales de 1999 ainsi que les assises fiscales de l'année 2013 et les séries de réformes et d'aménagements fiscaux qui les ont suivies. 


\section{Méthodologie}

Le présent travail a pour objectif d'analyser l'évolution du système fiscal marocain de l'indépendance en 1956 à la mise en œuvre de la réforme fiscale des années 1980 afin d'en tirer les enseignements et pouvoir se prononcer sur ses évolutions futures. Pour ce faire, et en guise de mise en contexte, est présenté le système fiscal de l'indépendance, sa structure et ses caractéristiques. Il est aussi évalué au regard de ses performances et ses contreperformances. Ensuite la réforme fiscale des années 1980, grand tournant de l'évolution du système fiscal marocain, est analysée au vu de son cadre général, et de son bilan global. Nous arrivons à la conclusion que le bilan de cette réforme est mitigé, des défaillances persistent malgré les avances accomplies.

\section{Formation Du Système Fiscal D'avant La Réforme Des Années 1980}

Après l'indépendance en 1956, les pouvoirs publics se sont contentés de reconduire le système fiscal légué par le protectorat français. Pourtant, la puissance coloniale s'est limitée à greffer au Maroc son propre modèle. Le système hérité visait plus la collecte des ressources financières que le développement économique et social. Dans les trois décennies qui allaient suivre l'indépendance, la structure du système légué par l'administration coloniale n'a guère fondamentalement changé, il a gardé son armature et ses structures, se perpétuant au prix de quelques aménagements plus ou moins significatifs et - surtout - de nombreux relèvements des taux d'imposition (Akesbi, 1994).

Le système fiscal hérité du protectorat reposait sur une large prédominance des impôts indirects, pourtant il comprenait cinq composantes (Akesbi, 1994) :

$1^{\circ}$ une taxe sur le chiffre d'affaires à caractère général et largement cumulatif,

$2^{\circ}$ des taxes spécifiques à la consommation sur des produits particuliers (tabac, pétrole, huile, sucre ...),

$3^{\circ}$ des droits de douane, plus axés sur les droits à l'importation que sur les droits à l'exportation, avec naturellement des privilèges particuliers en faveur des échanges avec la métropole,

$4^{\circ}$ des impôts directs cédulaires à caractère réel et indiciaire créés progressivement pour mettre à contribution des sources de revenus déjà existantes ou apparues avec la croissance des secteurs " modernes" de l'économie : tertib, patente, impôt sur les bénéfices professionnels, prélèvement sur les traitements et les salaires, taxe urbaine ...

$5^{\circ}$ divers droits d'enregistrement et de timbre à assimiler aux impôts indirects dans la mesure où ils portent pour l'essentiel sur des droits perçus lors des transactions immobilières et des droits de timbre. 
Le Maroc a hérité ce système d'imposition malgré ses défauts : complexité, incohérence, injustice, cédularité excessive, gestion coûteuse et déséquilibres...Depuis l'indépendance, les aménagements et "réformes" fiscaux se sont succédés sans que le système fiscal hérité évolue nettement. Ils ont concerné la fiscalité douanière, les taxes intérieures à la consommation (TIC), la taxe d'édilité et la vignette automobile. Le tarif douanier a été réorganisé en 1957. Un nouveau régime avec des tarifs différenciés destiné à augmenter les recettes fiscales et à protéger l'industrie locale naissante s'est substitué à la taxe uniforme de $12,5 \%$ applicable tout au long du protectorat. Des taxes d'exportation ont été instituées sur les minerais, les agrumes et les autres produits destinés à l'exportation et les taxes intérieures de consommation sur les vins, alcools et pétrole ont été augmentées pour accroître les ressources indirectes (El Ktiri, 1979). De même, ont été instituées en 1956 la taxe d'édilité en remplacement de la taxe municipale d'entretien et d'enlèvement des ordures ménagères et en 1957 la vignette qui frappe les véhicules automobiles (El Ktiri, 1979).

La réforme fiscale engagée en 1961 n'a véritablement modifié que l'imposition des revenus agricoles. Le tertib entré en application en 1915 et dérivé de la Zakat a été supprimé par le discours royal prononcé le 19 juin 1961. Il fut remplacé par l'impôt agricole institué par le dahir $\mathrm{n}^{\circ} 1.61 .438 \mathrm{du}$ 30 décembre 1961 pour remédier aux insuffisances du tertib et adapter la fiscalité agricole aux exigences du développement économiques et social du pays (Bensalah Zemrani, 1982). A cet effet, il fut assis non sur le revenu réel mais sur le revenu virtuel fondé sur les terrains à usage agricole et arboricole. Aussi, le tarif de l'impôt agricole a conduit à exonérer une fraction importante des agriculteurs en relevant le seuil d'imposition (90\% environ). La tranche du revenu allant de 0 à $1400 \mathrm{DH}$ a été exonérée avec application d'un tarif progressif allant de $8 \%$ à $20 \%$.

L'année 1962 a connu d'autres aménagements fiscaux : création de la zone franche de Tanger avec son régime fiscal autonome et la publication du dahir $n^{\circ} 1-60-121$ du 23 mars 1962 relatif aux taxes communales. Et bien avant, les pouvoirs publics, soucieux de promouvoir l'investissement privé, ont promulgué les codes des investissements de 1958 et 1960.

La crise financière de 1964 a amené les autorités marocaines à mettre en place un plan de redressement, axé sur l'accroissement des recettes et la diminution des dépenses publiques. A ce titre, sont intervenus de nombreux relèvements des barèmes de quelques impôts directs et surtout des droits et taxes à la consommation (taxes sur les produits et services (T.P.S) et taxes intérieures à la consommation (T.I.C)), les droits de douane et de timbre ont été sensiblement relevés, mais les barèmes du prélèvement sur les traitements et salaires (PTS), de l'impôt agricole (IA) et de l'impôt sur les bénéfices professionnels (IBP) ont été également plus on moins alourdis (El Ktiri et 
Akesbi, 1987). Privilégiant la quantité sur la qualité, le plan de redressement de 1964 a permis le retour à l'équilibre budgétaire mais n'a pas amélioré la qualité du système fiscal en vigueur (El Ktiri et Akesbi, 1987). L'étude prospective des finances publiques menée par les auteurs du plan quinquennal 1968-1972 a conforté ce constat. Les planificateurs ont constaté que le système fiscal marocain est très peu progressif et ont repris à cet effet à leur compte certaines recommandations d'un projet de réforme fiscale globale élaboré en 1967 par un expert français (El Ktiri et Akesbi, 1987). Ils ont recommandé trois séries d'action (Division de la Coordination économique et du plan, 1968):

-amélioration de la production du système fiscal par la simplification de certains barèmes et procédures et par l'institution de certains contrôles (notamment pour les factures),

-aménagements fiscaux : réaménagement des bases imposables, limitation des réductions,

-institution de nouveaux impôts: les planificateurs ont pensé aux impôts suivants : impôt sur les successions, imposition des plus-values réalisées sur les terrains à bâtir, taxe spéciale sur les plus-values d'immeubles, impôt sur les plus-values des terres agricoles irriguées.

L'impôt sur les successions recommandé par les planificateurs a été institué en 1968 mais a été aussitôt supprimé au motif d'être contraire à la Chariâa islamique (Akesbi, 1989). De même, dans le cadre de la loi de finances pour l'année 1971, le gouvernement a présenté au parlement un projet de réforme de l'impôt agricole en raison de sa modicité et de son rendement faible. Mais les pouvoirs publics vont abandonner ce projet en raison de l'opposition des gros agriculteurs.

La loi de finances pour l'année 1972 a institué la contribution complémentaire sur le revenu des personnes physiques. Il s'agit d'un premier pas vers la mise en place d'un impôt général sur le revenu. La contribution complémentaire est un impôt de superposition mis en place pour appréhender la totalité des revenus d'un même contribuable qu'ils soient soumis où non à un impôt cédulaire, les profits immobiliers compris. L'objectif recherché, lors de son institution, est d'atteindre les revenus échappant aux impôts cédulaires.

D'autre part, pour appréhender les revenus du capital, ont été instituées en 1973, la taxe sur les produits des actions, parts sociales et revenus assimilés (TPA) et la taxe sur les produits de placement à revenu fixe (TPPRF). Ces taxes ne constituaient pas des impôts spécifiques mais des précomptes à la source imputables sur le montant de la contribution complémentaire. Toutefois, en juillet de la même année 1973, la TPPRF a été supprimée de crainte de son impact négatif sur l'épargne. Aussi, pour promouvoir les exportations et relancer l'investissement, l'année 1973 a connu l'entrée en 
vigueur de divers régimes économiques en douane et la promulgation de six nouveaux codes des investissements le 13 août 1973 en remplacement du code des investissements de 1960.

\section{Report Indu De La Réforme Fiscale}

Avec le lancement du plan de développement économique et social 1973-1977, la question de la réforme fiscale est revenue à l'ordre du jour. Les auteurs du plan quinquennal ont estimé que le système fiscal en vigueur peut être considéré comme satisfaisant du point de vue des recettes brutes puisqu'il permet de prélever 20\% environ de la PIB. Mais sur le plan qualitatif, il n'est pas équitable puisque les impôts directs n'y représentent que moins $20 \%$, tandis que les impôts indirects et les taxes à la consommation atteignent $45 \%$ du total (Direction du Plan et du développement, 1973). Afin de remédier à ces insuffisances et aboutir à un système fiscal simple, les planificateurs ont proposé les axes de réforme suivants (Direction du Plan et du développement, 1973):

-regroupement des multiples impôts directs, leurs taux et assiettes en deux catégories : un impôt sur les revenus des personnes physiques et un impôt sur les sociétés,

-réaménagement de l'impôt agricole en vue de l'amélioration de son rendement et soumission des sociétés agricoles à l'impôt sur les sociétés,

-transformation de la taxe urbaine en un impôt foncier et élargissement de son assiette,

-réorganisation de la taxe sur les produits et services en un système de la taxe sur la valeur ajoutée avec abaissement des taux sur les produits de grande consommation.

Mais les propositions des planificateurs n'ont pas été mises en œuvre. Les pouvoirs publics se sont passés de la réforme fiscale en raison de la hausse subite des cours de phosphates. Néanmoins, en 1978, avec la dégradation des équilibres financiers, les pouvoirs publics vont solliciter le Fonds Monétaire International (FMI) pour la réforme du système fiscal marocain. La mission envoyée à cet effet a élaboré un projet de réforme assez détaillé axé autour des éléments suivants : abaissement des barrières douanières, substitution de la TVA aux taxes sur les produits et services, substitution d'un IGR aux différents impôts cédulaires, substitution de l'IS à l'impôt sur les bénéfices professionnels (IBP), institution d'un impôt foncier et d'un impôt assis sur les terres agricoles, l'extension des TIC à d'autres produits, et la refonte de l'impôt des patentes dans l'impôt sur le revenu des sociétés (El Ktiri et Akesbi, 1987).

Le plan triennal 1978-1980 est allé dans le même sens. Les planificateurs ont réitéré leur appel à la réforme fiscale et ont constaté que les orientations préconisées en la matière dans le cadre du plan 1973-1977 n'ont pas été toutes suivies (Direction du Plan et du développement, 1973). Mais les 
pouvoirs publics vont reporter de nouveau l'échéance de la réforme fiscale. La phase 1978-1983 a enregistré l'hésitation des pouvoirs publics entre la poursuite de la relance économique et l'engagement d'une politique de stabilisation et de maintien des équilibres macro-économiques. Cette hésitation a coûté cher à l'économie nationale, les équilibres macroéconomiques se sont dégradés et une grave crise financière s'est installée. Le niveau des réserves de change était inquiétant, il ne dépassait pas l'équivalent de 18 jours d'importation en 1982 contre deux mois au début des années soixante-dix. A son tour, le profil de la dette extérieure s'est nettement détérioré. Le rapport encours de la dette / PIB est passé de $55 \%$ en 1980 à 80 $\%$ en 1981 et à $97 \%$ en 1983 où encore $110 \%$ en 1984 et $123 \%$ en 1985 . Devant cette situation, les responsables seront contraints d'adopter et mettre en œuvre le programme de stabilisation macro-économique et d'ajustement structurel de l'économie marocaine (PAS).

Au cours de la phase 1978-1982, les pouvoirs publics se sont passés d'une réforme fiscale mais ont continué à apporter des réaménagements et des retouches au système fiscal en vigueur. Ainsi, devant le développement croissant du secteur immobilier et les difficultés des finances publiques, ils ont pris en 1978 une série de mesures pour réformer la fiscalité immobilière :

-personnalisation de la taxe urbaine par l'application d'un nouveau barème progressif. Ce barème est venu se substituer au taux proportionnel uniforme de 13,5\% applicable depuis 1961.

-institution de la taxe sur les profits immobiliers (T.P.I) réalisés à l'occasion de la cession de biens immeubles où des droits réels immobiliers. La nouvelle taxe comporte un barème progressif variant en fonction de la durée de détention du bien cédé. Mais ce barème a été annulé et remplacé dans le cadre de la loi de finances pour l'année 1980 par un taux proportionnel de $15 \%$. Avec l'institution de la T.P.I, les revenus immobiliers sont devenus exclus de la contribution complémentaire.

-institution de l'impôt sur les terrains urbains comportant une taxe unique perçue sur les terrains inclus dans les périmètres urbains par suite de l'extension de ces périmètres et une taxe annuelle sur les terrains urbains non bâtis. Mais l'impôt sur les terrains urbains qui devrait entrer en application à partir du $1^{\text {er }}$ janvier 1981 est resté gelé pour être supprimé purement et simplement en 1983.

La réforme de la fiscalité immobilière de 1978 a été couronnée par la promulgation du code des investissements immobiliers de 1981 qui a rendu caduques les mesures précitées et a fait bénéficier les promoteurs immobiliers d'une quasi-immunité fiscale (El Ktiri et Akesbi, 1987). 


\section{Le Système Fiscal D’avant La Réforme Des Années 1980 : Un Système Aux Multiples Défaillances}

Les réaménagements successifs et retouches introduits progressivement depuis 1'indépendance ont abouti à un système fiscal complexe. Ce système accumulait défaillances qualitatives et financières qui rendaient inévitable une réforme fiscale profonde et continue.

\section{Architecture du système fiscal d'avant la réforme}

Le système fiscal d'avant la réforme était constitué d'une mosaïque d'impôts et taxes :

-11 impôts directs : l'impôt agricole, la patente, la taxe de licence sur les débits de boissons, le prélèvement sur les traitements et les salaires, la taxe urbaine et le taxe d'édilité, la taxe sur les profits immobiliers, la taxe sur les produits des actions, parts sociales et revenus assimilés, la contribution complémentaire sur le revenu des personnes physiques, la participation à la solidarité nationale et l'impôt sur les terrains urbains,

-une multitude d'impôts indirects : deux taxes sur le chiffre d'affaires (la taxe sur les produits et la taxe sur les services (TPS)) et quatre groupes de taxes intérieures de consommation : l'impôt sur le tabac, la taxe sur les vins et alcools, la taxe sur les produits pétroliers, la taxe sur le sucre, la taxe sur les spectacles, la taxe sur les allumettes, le timbre de quittance, les droits d'essai et de garantie des matières d'or, d'argent et de platine ...

-des droits de douanes plus axés sur les droits à l'importation que sur les droits à l'exportation. Les importations étaient soumises à quatre impôts : le droit d'importation, la taxe spéciale, le timbre douanier et la taxe sur les produits et les services. Les exportations étaient soumises à la taxe statistique à l'exportation, la taxe de contrôle technique à l'exportation pour les produits de l'artisanat, la taxe d'exportation des produits agricoles et agro-industriels et au prélèvement sur les exportations de produits miniers.

Mais les réaménagements successifs et retouches fiscaux introduits n'ont pas épargné au Maroc la grave crise financière de 1982. Cette voie a été alors épuisée. L'année 1982 a constitué un tournant dans l'évolution du système fiscal marocain. La crise financière de 1982 a précipité la grande réforme fiscale de la décennie 1980. L'Etat s'est trouvé obligé de solliciter le rééchelonnement de la dette publique et par conséquent de se soumettre aux directives du Fonds Monétaire International (FMI) et de la Banque Mondiale. Le plan d'ajustement structurel (P.A.S) alors mis en place a intégré un plan d'assainissement des finances publiques. La réforme fiscale introduite au milieu des années 1980 s'inscrit dans ce cadre. Elle a constitué une partie majeure du P.A.S. Le processus de réforme fiscale va s'enclencher avec l'adoption de la loi-cadre relative à la réforme fiscale votée en 1982 et promulguée en 1984. 


\section{Limites du système fiscal d'avant la réforme}

La réforme fiscale de 1984 est conçue pour rationaliser et la simplifier le système fiscal en vigueur et remédier à ses insuffisances. Nous présentons dans ce qui suit les principales caractéristiques de ce système fiscal :

-cédulaire, composé d'une mosaïque d'impôts et taxes, le système fiscal d'avant la réforme apparaissait lourd et complexe. La cédularité se traduisait par la multiplicité des tâches matérielles et l'enchevêtrement des procédures. Le contribuable se voyait imposer de nombreuses et formalités, et l'administration se heurtait à des difficultés de coordination de ses compartiments techniques cloisonnés et la multiplicité des dossiers fiscaux : un dossier par contribuable et par catégorie de revenu (Direction des Impôts, 1982).

-cédulaire, constitué de composantes disparates, le système fiscal d'avant la réforme était largement inégalitaire. Les sources de l'inégalité étaient multiples: disparités des taux d'imposition pour des tranches de revenus identiques, disparités dans le découpage des tranches, seuils d'exonération fixés à des niveaux différents $(6000 \mathrm{DH}$ pour les salariés, $3000 \mathrm{DH}$ pour les professionnels et les rentiers, $1400 \mathrm{DH}$ pour les agriculteurs), déductions pour charges de famille comportant de nombreuses distorsions, disparités dans la détermination des bases imposables (la base imposable est déterminée de manière spécifique pour chaque catégorie de revenu), inégalité résultant du fractionnement du revenu (Direction des Impôts,1982). L'inégalité résultant du fractionnement du revenu pénalise le contribuable qui dispose d'une seule catégorie de revenus et avantage le contribuable dont les revenus sont diversifiés.

-bien que cédulaire et constitué d'une multitude de composantes disparates, le système fiscal d'avant la réforme ignorait des sources de revenus et de richesses importantes alors que des catégories de revenus (salaires, revenus professionnels et locatifs) subissaient une cascade d'impositions : absence d'un impôt sur le capital et sur les produits de placements à revenus fixes, exonération prolongée des revenus agricoles, exonérations fiscales importantes accordées par les codes des investissements (El Ktiri et Akesbi, 1987).

-le système fiscal d'avant la réforme avait le mérite de permettre la collecte de ressources équivalent au cinquième environ du produit intérieur brut (PIB) (Akesbi, 1986). Plus précisément, le rapport recettes fiscales/PIB (ou pression fiscale) a évolué globalement vers la hausse passant de $15,9 \%$ en 1968 à $19,5 \%$ en 1975 et à $20,1 \%$ en 1982 . Cette progression importante a été enregistrée durant le plan 1973-1977 : le taux de pression fiscale n'a été que de $15,1 \%$ en 1975 et $14,7 \%$ en 1974 . Mais la progression importante de la pression fiscale ne doit pas occulter le fait qu'à la veille de l'application du P.A.S, les recettes fiscales arrivaient à peine à couvrir la moitié des dépenses 
publiques et que le taux de couverture des dépenses publiques par les recettes fiscales a nettement baissé passant de $74,8 \%$ en 1973 à $62,5 \%$ en $1978,58,2 \%$ en 1979 et $55 \%$ en 1982.

-fondée essentiellement sur les impôts indirects, la structure des recettes fiscales était largement déséquilibrée. Ainsi, en 1985, la part des impôts indirects y compris les droits de douane, dans les recettes fiscales totales, s'élevait à $65 \%$ alors que la part des impôts directs ne dépassait pas $25,7 \%$ contre $37,7 \%$ en 1975 . Bien plus, à l'intérieur de chaque catégorie d'impôts, deux ou trois impôts rapportaient l'essentiel des recettes. A titre d'exemple en 1985, l'Impôt sur les bénéfices professionnels (IBP) et le Prélèvement sur les traitements et les salaires (PTS) ont rapporté 76\% des recettes fiscales provenant des impôts directs, la taxe sur les produits et les services (TPS) a rapporté $75 \%$ des recettes provenant des impôts indirects et la taxe spéciale à l'importation ainsi que les droits à l'importation ont rapporté $93 \%$ des recettes provenant de la fiscalité douanière.

-de par sa structure déséquilibrée des recettes fiscales, le système fiscal d'avant la réforme se traduisait par des limites sur le plan social, économique et financier: injustice sociale, forte dépendance de la conjoncture économique, aggravation des déficits extérieurs, accent mis sur la consommation de masse et les revenus modestes.

\section{L'inévitable Réforme Fiscale Des Années 1980 : Cadre Général}

Les défaillances du système fiscal étaient perceptibles à tous et faisaient l'unanimité. Les autorités marocaines, malgré le report successif d'une réforme inévitable, ont fini par reconnaître les carences du système fiscal en place et la nécessité de sa réforme. La promulgation, le 23 avril 1984, de la loi-cadre $n^{\circ} 3-83$ relatives à la réforme fiscale adoptée par la chambre des représentants le 20 Décembre 1984 est intervenue dans ce sens. Cette loi a fixé les objectifs fondamentaux de la réforme fiscale et tracé son cadre général. L'article premier de la loi n ${ }^{\circ} 3-83$ a délimité le champ de la réforme fiscale en stipulant qu'elle porte sur les impôts directs assis sur les revenus des personnes physiques et morales, la taxe sur les produits et la taxe sur les services. Le titre III de ladite loi a ajouté la réforme de la fiscalité des collectivités locales. Ainsi, se trouvent exclus, en principe, du champ de la réforme fiscale, la fiscalité douanière, les taxes intérieures de consommation et les droits d'enregistrement et de timbre. L'article premier de la loi n ${ }^{\circ} 3-83$ a exclu aussi la réforme des codes des investissements.

Mais les responsables marocains ne se sont pas limités au cadre tracé par la loi-cadre. Au total, la réforme fiscale engagée, depuis le milieu des années 1980 a abouti à la réforme de la fiscalité douanière, la réforme des codes des investissements en 1988, la réforme de la fiscalité locale, la substitution de la T.V.A à la taxe sur les produits et les services (TPS) en 1986, 
la substitution de l'impôt sur les sociétés (IS) à l'IBP en 1987 et l'institution de l'Impôt Général sur le Revenu (IGR) en 1990. L'article premier de la loicadre relative à la réforme fiscale en a fixé aussi les objectifs :

-corriger les disparités du système fiscal en vigueur,

-prévenir et supprimer la fraude et l'évasion fiscales dans le respect des procédures légales garantissant les droits des contribuables,

-promouvoir et consolider les finances des collectivités locales,

-encourager les investissements. La réforme fiscale ne devrait pas porter atteinte aux mesures tendant à encourager les investissements.

Ces objectifs suscitent, néanmoins, des remarques. La réforme fiscale constitue une partie intégrante du programme d'ajustement structurel et par conséquent devrait permettre de rétablir les équilibres économiques et financiers. Il s'ensuit que le principal déterminant de la réforme fiscale est d'abord financier: collecte des ressources nécessaires au rétablissement de l'équilibre budgétaire et remboursement de la dette (Akesbi, 1994). Aussi, de par son contenu, la réforme fiscale a visé la modernisation et la simplification du système fiscal en vigueur. Elle s'est traduite, en effet, par le passage d'un système cédulaire complexe et caractérisé par des inégalités flagrantes, des déséquilibres et des incohérences à un système d'imposition moderne et synthétique.

Ainsi, l'IGR s'est substitué à six impôts cédulaires : le prélèvement sur les traitements et salaires, la taxe urbaine, pour les revenus locatifs, l'impôt sur les bénéfices professionnels, l'impôt agricole, la contribution complémentaire sur le revenu des personnes physiques et la taxe sur les produits des actions, parts sociales et revenus assimilés. L'IS a remplacé trois impôts cédulaires : l'impôt sur les bénéfices professionnels, l'impôt agricole et la taxe urbaine pour les revenus locatifs. La TVA a pris la place de la taxe sur les produits et la taxe sur les services. A cela, s'ajoutent l'amélioration des procédures fiscales, la réduction des taux d'imposition, les garanties supplémentaires accordées au contribuable et les diverses mesures d'accompagnement prises (restructuration de l'administration fiscale, recrutement et formation du personnel, informatisation progressive des services fiscaux, effort d'information du public...).

La réforme fiscale initiée au milieu des années 1980 s'est fixé un autre objectif : la consolidation des finances des collectivités locales. Dans ce sens, la loi-cadre $n^{\circ} 3-83$ a prévu l'affectation aux budgets des collectivités locales du produit de l'impôt des patentes et de la taxe urbaine après défalcation d'un pourcentage de $10 \%$ pour couvrir les frais d'assiette et de recouvrement et une part du produit de la T.V.A s'élevant à $30 \%$. Elle a prévu, aussi, la création de nouvelles taxes pour augmenter les ressources des collectivités locales. Et c'est dans cette perspective qu'a été promulguée la loi n $30-89$ relative à la fiscalité des collectivités locales et de leurs groupements s'est substituée au dahir du 23 
mars 1962 qui régissait la fiscalité locale depuis le début de la décennie 60 et à d'autres textes législatifs et réglementaires dispersés et difficiles à connaitre par les gestionnaires communaux (Mamoune, 2001). La nouvelle loi a mis fin à cette dispersion et regroupé, en un seul texte, tous les textes relatifs à la fiscalité locale en vue d'une harmonisation législative (Mamoune, 2001).

La loi $\mathrm{n}^{\circ} 30-89$ a reconduit plusieurs taxes existantes et créé de nouveaux impôts et taxes portant sur les secteurs de l'immobilier (taxes sur les terrains urbains non bâtis), de l'automobile et des transports (taxe sur les permis de conduire, taxe sur les motocyclettes, taxe sur les licences de taxis et de transport public des voyageurs ...), des loisirs et de détente (taxe sur les permis de chasse, taxe de séjour ...) et divers autres secteurs.

Mais la loi n ${ }^{\circ}$ 30-89 se compose d'une multiplicité des impôts et taxes : au total, elle comporte 49 impôts, taxes, droits et redevances locaux répartis entre les communes urbaines et rurales, provinces et préfectures, communautés urbaines (conseils de villes) et régions. Aussi , une concurrence et double emploi entre fiscalité d'Etat et fiscalité locale est constatée : cas de la participation à la solidarité Nationale (PSN) sur terrains non bâtis et de la taxe sur les terrains urbains non bâtis, cas des taxe de séjour et taxe de promotion touristique, taxe sur les débits de boissons alcooliques et de la taxe de licence sur les débits de boissons. La loi $\mathrm{n}^{\circ}$ 30-89 se caractérisait également par le caractère anti-économique de certains impôts et taxes - cas de l'impôt des patentes et de la taxe urbaine à titre d'exemple qui frappent l'investissement- et intégrait des redevances qui ne répondent pas aux critères de définition des impôts et taxes.

L'entrée en application de la loi $\mathrm{n}^{\circ}$ 30-89 relative à la fiscalité des collectivités locales et de leurs groupements a montré d'autres insuffisances :

-précarité de la matière imposable et existence de taxes nuisibles telles que la taxe pour fermeture tardive et ouverture matinale,

-coût de gestion élevé de certains impôts et redevances dépassant dans certains cas les recettes qu'ils génèrent et faible rendement financier pour la totalité du système fiscal local comme en témoigne le déficit chronique des collectivités locales,

-défaillance du système de recouvrement qui fait perdre aux communes une grande part de leurs recettes. En effet, les restes à recouvrer représentent $64 \%$ des recettes et l'essentiel de ces impayés reste irrécupérable et se chiffre pour la seule année 2002 à 5 milliards de DH (Mamoune, 2001).

En dépit de la nouvelle réforme de la fiscalité locale, les collectivités locales continuaient de dépendre dans leur financement de l'Etat puisque les recettes provenant de la fiscalité locale proprement dite (fiscalité gérée par les collectivités locales) ne représente que $24 \%$ des ressources financières des communes en 2005 (Direction des Finances Locales, 2008). Tandis que les transferts de l'Etat composés du produit de la TVA, de l'IS et de l'IGR et le 
produit de la fiscalité gérée par la Direction Générale des Impôts (taxe urbaine, taxe d'édilité et impôts des patentes) assurent respectivement $53.3 \%$ et $16.9 \%$ des ressources globales des collectivités locales (Direction des Finances Locales, 2008). Face à ces insuffisances, les pouvoirs publics ont procédé, de nouveau, à la réforme de la fiscalité locale et adopté la loi $\mathrm{n}^{\circ} 47-06$ relative à la fiscalité des collectivités locales à fin 2007 en remplacement de la loi n 30 89.

\section{La Réforme Fiscale Des Années 1980 : Un Bilan Mitigé}

Le cadre général de la réforme fiscale engagée depuis 1984 étant précisé. Pour mieux en saisir la portée, il convient de dresser un bilan de cette réforme à commencer par les apports et les limites des trois impôts majeurs issus de la réforme fiscale à savoir la T.V.A, l'I.S et l'I.G.R ${ }^{(6)}$. Aussi, la réforme fiscale des années 1980 si elle a amélioré les recettes fiscales, elle ne s'est pas accompagnée de l'amélioration de leur structure.

\section{Apports et limites des impôts synthétiques : T.V.A, I.S et I.G.R}

Les apports de la T.V.A comme ses limites sont nombreux. A mettre à l'actif de la T.V.A, la fusion en une seule taxe de deux taxes sur le chiffre d'affaires, l'extension de son champ d'application au commerce de gros puis au commerce de détail, la généralisation et la simplification du système de déduction et partant la réduction des coûts et l'incitation à l'investissement, l'obligation de tenir une comptabilité régulière et de la facturation et la modernisation et la rationalisation conséquentes de la gestion des entreprises assujetties, l'aménagement des taux d'imposition, l'extension progressive de la base imposable et la réduction des taux d'imposition, l'encouragement des exportations soumises au taux de $0 \%$, les garanties supplémentaires accordées au contribuable...

En parallèle de ces apports, la T.V.A présente bien des limites telles que la multiplicité des taux et des exonérations, les exceptions qui limitent le système de déduction, l'inélasticité de la taxe due à son mode de calcul fondé sur l'encaissement et non la facturation, la lenteur au niveau des remboursements malgré les délais raccourcis, la procédure contentieuse lente, l'insuffisance des moyens de contrôle de l'administration face aux garanties importantes accordées aux assujettis, la non déductibilité de la T.V.A sur le carburant jusqu'en 2001, l'alourdissement des prix des biens et services de première nécessité en raison de leur exonération sans droit à déduction, la soumission à la T.V.A d'autres produits de large consommation, la non

(6) De par la multiplicité de ces apports et limites et la nature du présent travail, nous nous contentons de les citer brièvement. 
application saine de la T.V.A en raison de la structure économique dualiste du Maroc (secteur moderne, secteur traditionnel)...

Le second grand impôt majeur issu de la réforme fiscale introduite au milieu des années 1980 est l'impôt sur les sociétés. Les apports de l'IS sont multiples: institution de la cotisation minimale, paiement de l'impôt par quatre acomptes provisionnels, champ d'application plus large que l'IBP (intégration des revenus agricoles et locatifs), définition plus précise des opérations imposables et des charges déductibles, aménagement des taux d'imposition par la substitution d'un barème à un taux unique à un barème progressif, baisse de la charge fiscale par la réduction progressive des taux d'imposition, substitution du paiement spontané à l'émission par voie de rôle, renforcement des garanties des contribuables, respect du principe de l'unicité du patrimoine et du résultat fiscal ...

Mais l'I.S n'est pas exempt de limites : gonflement des charges déductibles demeurées sans limitation, limitation du champ d'application par les privilèges accordés au secteur agricole, discrimination à l'égard des PME par l'institution du taux unique de l'IS et partant un traitement fiscal égal à l'égard de toutes les sociétés assujetties, nombre élevé de sociétés qui déclarent des déficits successifs tout en continuant à exister, insuffisance des moyens de contrôle de l'administration devant les garanties multiples accordées aux contribuables, quasi-stagnation de la part de l'IS dans les recettes fiscales totales (10,3\% en 1998 contre $9,2 \%$ en 1987) ...

Le dernier impôt majeur issu de la réforme fiscale est l'Impôt Général sur le Revenu (I.G.R) devenu l'Impôt sur le Revenu (IR) depuis la loi de finances 2006. L'IGR est un impôt synthétique, moderne, venu pour remédier aux insuffisances de l'ancien système cédulaire (dénivellement des seuils d'imposition, distorsions qui affectent les déductions pour charges de famille, disparité des barèmes d'imposition). Ses apports sont signifiants : globalisation du revenu, unification des déductions et des procédures d'imposition, application d'un même barème d'imposition, institution du régime optionnel de comptabilité simplifiée, institution d'une cotisation minimale pour les revenus professionnels, institution de la déduction forfaitaire des charges pour les revenus fonciers, amélioration des rapports contribuables - administration fiscale ...

Mais l'IGR recèle des insuffisances comme l'exonération continue des revenus agricoles jusqu'en $2013\left({ }^{7}\right)$, une composition hétérogène $\left({ }^{8}\right)$, la progressivité régressive du barème de l'I.G.R d'où allégement considérable de la charge fiscale pour les détenteurs de revenus élevés et resserrement du barème pour les revenus modestes, le traitement inégal des différents revenus

$\left({ }^{7}\right)$ Article 7 de la loi de finances $n^{\circ} 40-08$ pour l'année budgétaire 2009.

$\left({ }^{8}\right)$ L'IGR apparaît comme une addition des anciens impôts cédulaires puisque la base imposable pour chaque catégorie de revenu est déterminée de manière différente. 
quant aux possibilités de déduction offertes, l'appréhension avec rigueur surtout des revenus salariaux de par le mode de recouvrement spécifique à cette catégorie de revenus : la retenue à la source, l'existence de régimes conventionnels plus avantageux que le droit commun, l'imposition à des taux élevés des tranches de revenu supérieures à d'où impact négatif sur le recrutement des cadres supérieurs (ingénieurs, cadres...) surtout pour les PME, le nombre réduit des contribuables qui paient l'IGR représentant $43 \%$ seulement de la population active citadine et ceux versant effectivement un IGR représentant seulement 19\% de cette population, le faible nombre de contribuables effectifs soumis à l'I.G.R grevant les recettes fiscales et faisant peser une charge lourde sur les plus productifs, les déductions sur le revenu global imposable bénéficiant de par leur nature surtout aux détenteurs de hauts revenus ...

\section{Amélioration non négligeable des recettes fiscales}

Globalement, la réforme fiscale des années 1980 s'est accompagnée d'une amélioration des recettes fiscales. Le rapport recettes fiscales/PIB est passé de 20,1\% en 1982 à 20,4\% en 1988, 21,9\% en 1990 et 23,8\% en 1992 . Il a été de 19,4\% en moyenne sur la période 1980-1989, 21,1\% sur la période 1990-1995 et 9,4\% sur la période 1996-2003. Mais la hausse annuelle moyenne des recettes fiscales qui a été de 12,9\% sur la période 1980-1989 a baissé depuis la fin du P.A.S pour se situer à 7,1\% sur la période 1996-2003. Ce ralentissement s'explique par la baisse des droits de douane suite au démantèlement tarifaire : leur variation annuelle a été de 10,3\% sur la période 1980-1989, 5,7\% seulement sur la période 1990-1995 et négative sur la période 1996-2003 soit: $-1,3 \%$. Le rythme de croissance des recettes des impôts directs et des impôts indirects s'est ralenti aussi. Pour les impôts directs, il a été de 13,8\% sur la période 1980-1989, 7,7\% sur la période 1990-1995, et 9,4\% sur la période 1996-2003. Pour les impôts indirects, sur les mêmes périodes, il a été respectivement de $11,3 \%, 12,7 \%$ et $4,5 \%$.

De même, le rôle prééminent dans le financement du budget de l'Etat est dévolu certes aux recettes fiscales. Mais l'importance des recettes non fiscales s'est accrue aussi. Alors que la pression fiscale s'est relativement stabilisée (19,4\% devant la période 1980-1989, 22,1\% durant la période 19901995, 22,1\% durant la période 1996-2003), le rapport recettes non fiscales/ PIB a progressé passant de $2 \%$ durant la période $1980-1989$, à 2,8\% durant la période 1990-1995 et à 3,9\% durant la période 1996-2003. Cette progression est due à la croissance des recettes de la privatisation. Le rapport recettes privatisations/PIB a été de $0,9 \%$ en $1993,1 \%$ durant le premier semestre 1996 , $1,5 \%$ durant l'année budgétaire 1996-1997, négligeable jusqu'en 2001 où il a atteint $6,1 \%, 0,2 \%$ durant la période $1990-1995$ et 1,3\% durant la période 1996-2003. Mais les recettes de la privatisation ont nettement baissé depuis 
l'année 2003. Elles ont été nulles en 2008, 2009, 2010,2013 et 2015. Le rapport recettes de la privatisation/PIB est passé de $1.2 \%$ durant la période 2000-2007 à 0.1\% seulement durant la période 2008-2016.

\section{Une structure déséquilibrée des recettes fiscales}

L'amélioration des recettes fiscales ne s'est pas accompagnée d'une évolution de leur structure. Ainsi, la part des impôts indirects ( TVA, TIC, et autres) dans les recettes fiscales totales a baissé légèrement passant de $42.4 \%$ au titre de la période 1990-1997 à $41.2 \%$ au titre de la période 19982002 et à $38.5 \%$ au titre de la période 2003-2008. A contrario, la part des impôts directs s'est accrue progressivement. Elle est passée de $30.3 \%$ au titre de la période 1990-1997 à 36.2 \% au titre de la période 1998-2002 et à $44.4 \%$ au titre de la période 2003-2008.

A l'intérieur des impôts directs, l'essentiel des recettes est rapporté par l'IS et l'IGR : $96.62 \%$ durant la période $2003-2008$ et $77 \%$ durant la période 1996-2003 contre 76\% pour l'IBP et le P.T.S en 1985. Au niveau des impôts indirects, l'essentiel des recettes fiscales est rapporté par la TVA et les taxes intérieures de consommation (TIC). La T.V.A (à l'intérieur et à l'importation) a rapporté $46.69 \%$ des recettes provenant d'impôts indirects - au sens strict - au titre de la période 1990-1997, 49.27\% au titre de la période 1998-2002 et $62.85 \%$ au titre de la période 2003-2008 contre $75 \%$ pour la T.P.S en 1985. Au titre de ces mêmes périodes, les TIC ont rapporté respectivement $53.3 \%, 50.7 \%$ et $37.14 \%$. Les droits à l'importation et le prélèvement fiscal à l'importation rapportent la quasi-totalité des recettes douanières : $85.98 \%$ au titre de la période $2003-2008,59.76 \%$ au titre de la période 1998-2002, 99\% durant la période 1990-1995, 95\% durant la période 1980-1989, contre $93 \%$ en 1985 pour la taxe spéciale à l'importation et les droits à l'importation.

Les inégalités induites par le système fiscal marocain se manifestent aussi par la fiscalisation importante du facteur travail. Le taux marginal de l'IR a été toujours plus élevé que le taux de l'IS comme le montre le tableau suivant.

\begin{tabular}{|c|c|c|c|c|c|c|c|}
\hline & $\mathbf{1 9 8 6}$ & $\mathbf{1 9 8 8}$ & $\mathbf{1 9 9 0}$ & $\mathbf{1 9 9 3}$ & $\mathbf{1 9 9 4}$ & $\mathbf{1 9 9 6}$ & $\mathbf{1 9 9 9}$ \\
\hline Taux de l'IS & $45 \%$ & $40 \%$ & $40 \%$ & $38 \%$ & $36 \%$ & $35 \%$ & $35 \%$ \\
\hline $\begin{array}{c}\text { Taux marginal de } \\
\text { l'IR }\end{array}$ & - & - & $52 \%$ & $48 \%$ & $46 \%$ & $44 \%$ & $44 \%$ \\
\hline $\begin{array}{c}\text { Seuil imposable à } \\
\text { l'IR en DH }\end{array}$ & - & - & 12.000 & 15.000 & 18.000 & 18.000 & 20.000 \\
\hline
\end{tabular}

Tableau $\mathrm{n}^{\circ} 1$ : Evolution des taux d'imposition en matière d'IS et d'IR

Source : Lois de Finances 


\section{Conclusion}

De l'indépendance au milieu des années 1980, les pouvoirs publics ont choisi de maintenir le système fiscal greffé par le protectorat français malgré ses défaillances. Le report indu de la réforme fiscale et les déséquilibres macroéconomiques conséquents nous renvoie à un dysfonctionnement structurel et récurrent dans la conduite de l'action publique au Maroc : la lenteur dans la prise de décisions et l'engagement des réformes structurelles. Avec la mise en œuvre de la réforme fiscale depuis 1984, le système fiscal marocain a réalisé certes des avancées mais des limites liées notamment à l'architecture du système et sa structure ainsi qu'aux nouveaux impôts synthétiques persistent.

Mais le processus de réforme du système fiscal marocain va se poursuivre notamment à la suite des premières et secondes assises nationales sur la fiscalité tenues respectivement en 1999 et 2013. Au cours des années 2000, et en application des recommandations des assises de 1999, la fiscalité nationale a subi une série de réaménagements et réformes en vue de la simplification de la législation fiscale, l'élargissement de la base d'imposition et la réduction des taux d'imposition. Toutefois, ces réaménagements successifs et réformes n'ont pas éliminé toutes les défaillances de la fiscalité marocaine. Confronté au contexte de crise économique et aux déséquilibres macroéconomiques importants, le système fiscal marocain devient sujet d'une nouvelle réforme.

Les secondes assises nationales sur la fiscalité de 2013 ont identifié trois points d'amélioration affectant le système fiscal marocain (Ministère de 1'Economie et des Finances, 2013): 1-un important potentiel fiscal non exploité; inhérent à la faiblesse de la culture du civisme fiscal au sein de la société, 2- des distorsions économiques alimentées par la multiplication des dépenses fiscales, la nécessité de rétablir la neutralité de la TVA pour les entreprises et par le mode de calcul de l'impôt sur le revenu, 3-un manque de confiance des citoyens vis-à-vis du systèmes fiscal. A l'issue des travaux de ces assises, ont été présentées les différentes propositions de réforme formulées par les participants. Ces propositions, encore en cours d'application depuis 2014, concernent la législation fiscale, la lutte contre la fraude fiscale et l'informel, la fiscalité et la compétitivité économique, l'Administration fiscale et la fiscalité locale et régionale.

\section{References:}

1. Akesbi, N., (1994). La réforme fiscale de l'ajustement structurel au Maroc : portée et limites. (pp. 233-234 ; p. 236). in Bilan décennal du programme d'ajustement structurel et perspectives de l'économie marocaine. Rabat, Annales Marocaines d'Economie, $\mathrm{n}^{\circ}$ spécial (hors série). 
2. Akesbi, N., (1986). La politique financière du Maroc indépendant, (p. 184). Rabat, La Grande Encyclopédie du Maroc, Volume Economie et Finances.

3. Akesbi, N., (1989). La réforme fiscale au Maroc : une issue à la crise des finances publiques, (p. 223), in Hamdouch, B (Etudes réunies par), Les politiques de sortie de crise et relations Nord-Sud, Rabat, Editions SMER.

4. Bensalah Zemrani, A., (1982). La fiscalité face au développement économique et social du Maroc, (p. 53). Paris, L.G.D.J.

5. Direction de la planification, (1979). Plan de développement économique et social 1978-1980, Volume I, (p. 139). Rabat.

6. Direction des Finances Locales, (2008). Guide de la fiscalité locale, (p. 7). Direction Générale des Collectivités Locales, Ministère de l'Intérieur, Rabat.

7. Direction du Plan et du développement, (1973). Plan de développement économique et social 1973-1977, Volume I, (pp. 5253). Rabat.

8. Direction des Impôts, (1982). Note sur la réforme fiscale, (pp. 27-30). Document inédit, Rabat.

9. Division de la Coordination économique et du plan, (1968). Plan quinquennal 1968-1972, Volume I, (pp. 122-123) .Rabat.

10. El Ktiri, M., Akesbi, N., (1987). La réforme de la fiscalité marocaine à l'heure de l'ajustement structurel, (pp. 15-16, pp. 19-22).Rabat, Les Editions Toubkal.

11. El Ktiri M., (1979). Structures fiscales et structures économiques, le cas de l'économie marocaine, (p. 53). Casablanca, Les Editions Maghrébines.

12. Mamoune, A., (2001). La réforme de la fiscalité locale au Maroc, (p. 09). Mémoire pour l'obtention du DEA en Finances publiques, Université Paris I / Université Paris XI.

13. Ministère de l'Economie et des Finances, (2013). Recueil des travaux des Assises Nationales sur la Fiscalité, 29 et 30 avril 2013, (p. 9). Rabat, AZ Editions. 